\title{
Unterarmfrakturen und distale Radiusfrakturen bei Kindern
}

Britta Sundermann, Katharina Mörs, Johannes Frank, Ingo Marzi

\section{Einleitung}

Frakturen des Unterarms stellen im Kindesalter die häufigste Frakturlokalisation dar. Unterschieden werden dabei metaphysäre Wulst- oder Stauchungsfrakturen, rein metaphysäre Frakturen, Frakturen mit Beteiligung der Wachstumsfuge, Unterarmschaftfrakturen und Frakturen des proximalen Unterarms. Wichtig ist es, bei der Versorgung von Frakturen im Kindesalter mit einem minimalen diagnostischen und therapeutischen Aufwand ein optimales Behandlungsergebnis zu erreichen. Dabei sollten nicht nur die Kriterien der Instabilität, Dislokation und das Alter, sondern auch immer das enorme Korrekturpotenzial des kindlichen Knochens, besonders am distalen Unterarm, berücksichtigt werden. Andererseits ist es wichtig, dass durch Fehlwachstum auch keine vermeidbaren funktionellen Einschränkungen entstehen.

\section{Prävalenz}

Mit über $40 \%$ stellen Frakturen des Unterarms die am häufigsten vorkommende Verletzung des kindlichen Skeletts dar [1]. Weiter untergliedert überwiegt mit knapp $75 \%$ der Fälle dabei die Fraktur des distalen Unterarms, gefolgt von der Unterarmschaftfraktur mit 20\% und der Fraktur des proximalen Unterarms in 5\% der Fälle [2]. In einer von Li-La (Licht und Lachen für kranke Kinder e. V.) initiierten Studie konnte gezeigt werden, dass mit steigendem Alter die Frakturhäufigkeit stetig zunimmt und der Altersgipfel um das 13. Lebensjahr liegt [3]. Bei den am häufigsten im Kindesalter auftretenden distalen Unterarmfrakturen überwiegen die Wulst- oder Stauchungsbrüche mit 40\%, gefolgt von Fugenschaftfrakturen (Epiphysenlösungen) mit oder ohne Metaphysenbeteiligung (30\%), den kompletten Frakturen (20\%) und den selten vorkommenden metaphysären Biegungsbrüchen (sog. Grünholzfrakturen, 10\%) [4]. Ursache bzw. Unfallmechanismus ist meist der Sturz beim Sport oder Spielen auf den ausgestreckten Arm [5].

\section{Klassifikation}

Unterteilt werden können die einzelnen Frakturen nach der Lokalisation oder dem Frakturtyp [10].

\section{Lokalisation}

- proximales Drittel

- isolierte Radiusfraktur

- Fraktur beider Unterarmknochen

- Monteggia-Verletzung

- mittleres Drittel

- isolierte Radiusfraktur

- Fraktur beider Unterarmknochen

- Grünholzfraktur

- distales Drittel

- isolierte Radiusfraktur

- Fraktur beider Unterarmknochen

- Galeazzi-Verletzung

\section{Frakturtyp}

- geschlossene oder offene Fraktur

- metaphysäre Wulst- oder Stauchungsfrakturen

- rein metaphysäre Frakturen

- Frakturen mit Beteiligung der Wachstumsfuge

- Grünholzfrakturen

- komplette Unterarmfrakturen

- Trümmerfrakturen

Gängige Klassifikationen sind u.a. die AO-Kindertraumaklassifikation. Dabei wird an erster Stelle das betroffene Körperteil mit einer Ziffer beschrieben. Die 2. Ziffer beschreibt die Lokalisation der Fraktur am betroffenen Knochen (Epi-, Meta- oder Diaphyse, vgl. > Abb. 1 und 2) [6].

Eine weitere gängige Klassifikation ist die Li-La-Klassifikation. Hierbei wird die Fraktur grundsätzlich durch 5 Positionen beschrieben, die 6. Position stellt eine Ausnahme dar. Die Ziffern 1-3 dienen der genauen Kennzeichnung der Lokalisation der Fraktur. Die Ziffern 4 und 5 beinhalten die Beschreibung der Morphologie der Fraktur, die Ziffer 5 definiert den Dislokationsgrad. Die 6. Ziffer beschreibt den zweiten der paarigen Knochen (vgl. - Abb. 3) [13].

\section{Korrekturpotenzial - tolerable Fehlstellungen}

Wichtig bei der Behandlung von Unterarmfrakturen ist die Wahl der richtigen Therapie unter Berücksichtigung des enormen Korrekturpotenzials des wachsenden Knochens. Spontankorrekturen sind besonders im Bereich des distalen Handgelenks aufgrund der Nähe zur Wachs- 


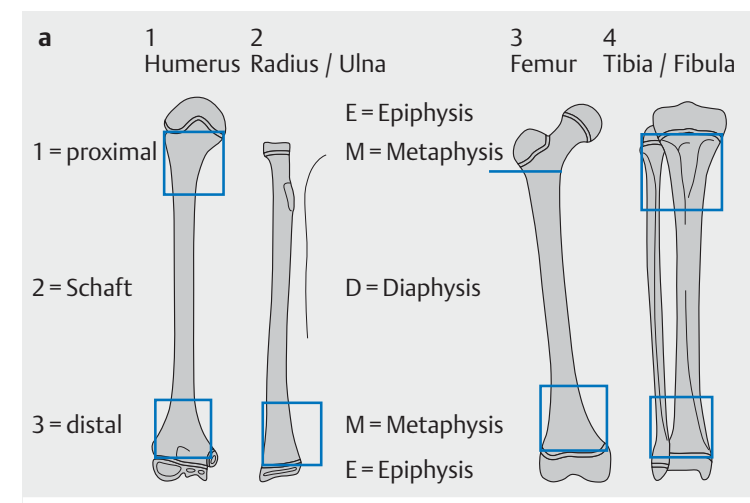

b $\quad E=$ Epiphysis
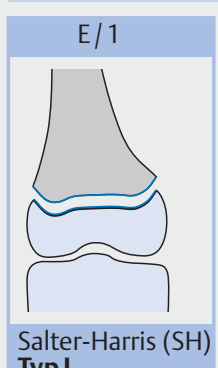

Typ I
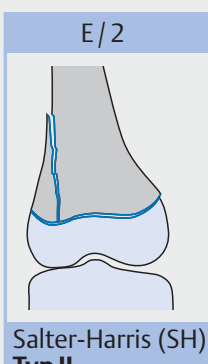

Typ II

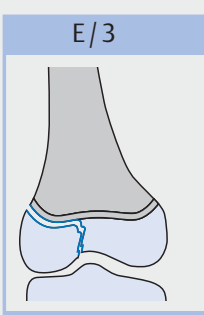

Salter-Harris (SH) Typ III

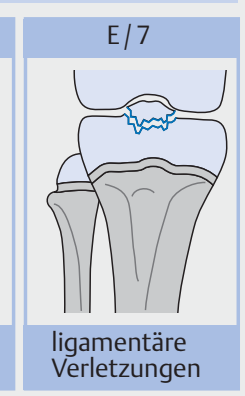

Typ IV

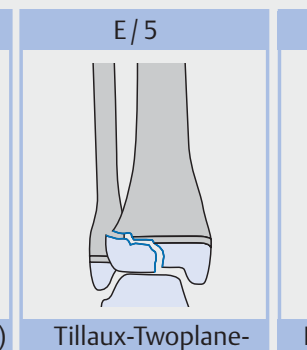

E/8

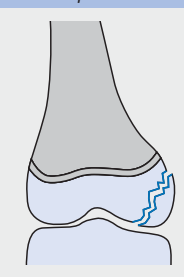

Flake Fractures Frakturen

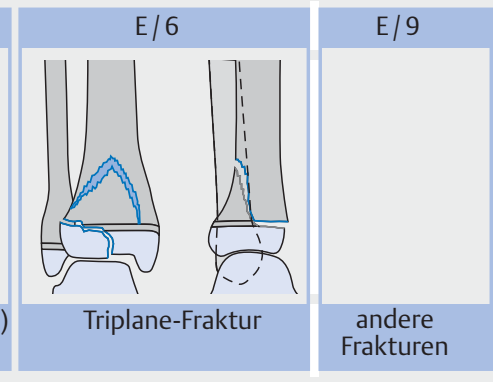

- Abb. 1 AO-Klassifikation der Frakturen langer Röhrenknochen und Subklassifikation der epiphysären Frakturen im Kindes- und Jugendalter by AO Foundation, Switzerland in SlongoT, AudigeL (2007) AO Pediatric comprehensive classification of long bone fractures (PCCF). AO Publishing, Davos a Quelle: Sommerfeldt D, Schneidmüller D. Klassifikation. In: Ruchholtz S, Wirtz D, Hrsg. Orthopädie und Unfallchirurgie essentials. 2. Aufl. Stuttgart: Thieme; 2012. b Quelle: Sommerfeldt D, Schneidmüller D. Allgemeines. In: Ruchholtz S, Wirtz D, Hrsg. Orthopädie und Unfallchirurgie essentials. 2. Aufl. Stuttgart: Thieme; 2012.

tumsfuge, dem hohen Anteil der distalen Fuge am Längenwachstum des Unterarms (80\%) sowie der Multidimensionalität der Beweglichkeit des Handgelenks enorm $[8,12]$. Studien haben gezeigt, dass eine Korrektur von Achsenfehlstellungen von $0,9^{\circ}$ pro Monat bzw. $10^{\circ}$ in einem Jahr möglich sind [9]. Weiterhin ist belegt worden, dass nicht nur Achsenfehlstellungen, sondern auch Seitzu-Seit-Verschiebungen bis zu einer vollen Schaftbreite gut korrigiert werden können. Wichtig an dieser Stelle zu erwähnen ist, dass eine Korrektur von Rotationsfehlern durch Wachstum nicht möglich ist $[9,10]$. Eine Altersgrenze des oben beschriebenen Korrekturpotenzials wird in der Literatur weiterhin diskutiert.

Üblicherweise wird die Grenze beim 10. Lebensjahr gezogen, eine Fehlstellung sollte ab dem Alter von 12 Jahren nicht mehr toleriert werden $[12,13]$.

Bei der Beurteilung ist es wichtig, die Lokalisation der Fraktur und ihr Korrekturpotenzial zu unterscheiden. Achsenabweichungen im Bereich des Unterarmschaftes werden nur bis zu $20^{\circ}$ korrigiert und machen hier häufig Probleme bei der Umwendbewegung, sodass Achsenabweichungen zwischen 10 und $20^{\circ}$ im Bereich des Unterarmschaftes nur bei kleinen Kindern akzeptiert werden sollten. Rotationsfehler dagegen werden hier nicht korrigiert, sodass bei höhergradigen Achsenabweichungen sowie Rotationsfehlern eine OP-Indikation besteht, um erhebliche Funktionseinschränkungen bei Pro- und Supination im Verlauf zu vermeiden. Eine Achsenabweichung über $10^{\circ}$ kann zu Störungen der Umwendbewegung führen und nicht belassen werden $[16,17]$. Sollte eine operative Korrektur nötig sein, so ist es wichtig, dass diese so früh wie möglich durchgeführt wird, um länger bestehende Funktionseinbußen zu verhindern, da diese auch nach Umstellung persistieren können und dadurch das Operationsergebnis beeinträchtigen können $[12,17]$.

Beim distalen Unterarm zeigt sich hingegen ein anderes Bild. Bis zum 10.-12. Lebensjahr weist dieser ein enormes Korrekturpotenzial auf, sodass Achsenabweichungen bis zu $30^{\circ}$ abfallend bis $10^{\circ}$ toleriert werden können. Bei Kindern, die das 12. Lebensjahr erreicht haben, besteht kaum noch eine Spontankorrektur. In diesen Fällen ist eine operative Therapie mit dem Ziel der achsengerechten Stellung anzustreben. Aufgrund der häufig starken Verunsicherung der Eltern ist eine ausführliche Aufklärung und enge Betreuung der Eltern bei z.T. deutlichen Fehlstellungen immens wichtig [11].

\section{Diagnostik}

Frakturen im Bereich des Unterarms werden in aller Regel bereits klinisch festgestellt. Die Anamnese, die Schonhaltung des Kindes und die Art des Sturzereignisses geben meist bereits den Hinweis darauf. 


\section{A einfache Fraktur}

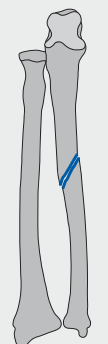

A 1

einfache Fraktur Ulna

ilfraktur

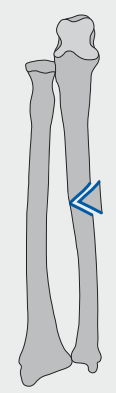

B 1

Keilfraktur Ulna

\section{C komplexe Fraktur}

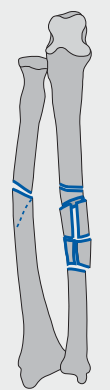

C 1

komplexe Fraktur Ulna, einfache Fraktur Radius

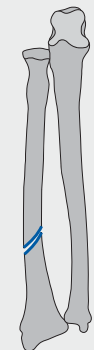

A 2 einfache Fraktur Radius

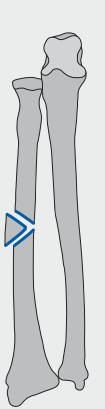

B 2 Keilfraktur Radius

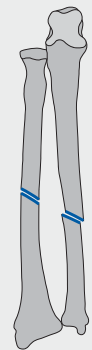

A 3

einfache Fraktur Ulna und Radius 
Li-La-Klassifikation

Version 2

\begin{tabular}{|c|c|c|c|c|c|}
\hline $\begin{array}{l}\text { 1. Stelle } \\
\text { Lokalisation } \\
\text { im Skelett: } \\
(1-4)\end{array}$ & $\begin{array}{l}\text { 2. Stelle } \\
\text { Lokalisation } \\
\text { im Knochen } \\
\text { (Segment): } \\
(1-3)\end{array}$ & $\begin{array}{l}\text { 3. Stelle } \\
\text { Morphologie: } \\
\text { - Gelenk (a) } \\
\text { - Schaft (s) }\end{array}$ & $\begin{array}{l}\text { 4. Stelle } \\
\text { Spezifizierungen } \\
\text { Morphologie: } \\
\text { - Gelenk }(1-5) \\
\text { - Schaft }(1-5)\end{array}$ & $\begin{array}{l}\text { 5. Stelle } \\
\text { Dislokationsausmass: } \\
\text { - undisloziert (0) } \\
\text { - tolerabel (1) } \\
\text { - nicht tolerabel (2) }\end{array}$ & $\begin{array}{l}\text { 6. Stelle } \\
\text { (Ausnahme) } \\
\text { paariger Knochen } \\
\text { nicht tragend } \\
\text { - Ulna }(\boldsymbol{U}) \\
\text { - Fibula }(\boldsymbol{F})\end{array}$ \\
\hline
\end{tabular}

- Im Schaftbereich wird mit proximal und distal jeweils die Metaphyse charakterisiert. Diese wird mit dem Quadrat über der zugehörigen Fuge definiert (Zirkelschlag von den Ecken der Epiphysenfuge aus).

- Gelenkverletzungen, die statistisch nicht ins

Gewicht fallen, werden an der jeweiligen

Lokalisation mit $5=$ andere klassifiziert

(proximaler Humerus, proximaler und distaler

Radius, proximale und distale Ulna sowie proximales Femur).

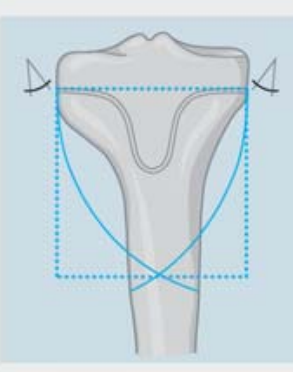

- Bei paarigen Knochen wird jeweils der haupttragende Knochen klassifiziert: Radius oder Tibia. Soll der Gegenknochen klassifiziert werden, wird an 6 . Stelle das $\mathbf{U}$ bzw. F eingefügt (s. z. B. Gelenkfrakturen Olekranon).

- Abb. 3 Li-La-Klassifikation von Frakturen im Wachstumsalter. Quelle: von Laer L. Li-La-Frakturklassifikation Version 2. In: von Laer L, Hrsg. Das verletzte Kind. Stuttgart: Thieme; 2007.

Eine operative Therapie ist indiziert bei einer Achsenabweichung $>10^{\circ}$ oder bei einer kompletten oder dislozierten Unterarmschaftfraktur, auch bei geringer Fehlstellung, aufgrund der Instabilität und des möglichen weiteren Fehlwachstums. Das Verfahren beinhaltet, wenn notwendig, ein Überbrechen der Gegenkortikalis und eine Stabilisierung mittels elastisch stabiler Marknagelung ( $\mathbf{A b b} . \mathbf{4})$.

Dabei werden elastische Federnägel der Größe 2,03,0 mm in den Knochen eingebracht. Die Implantatgröße sollte ein Drittel des Markraumdurchmessers betragen. Es wird sowohl ein radialer Zugang, der ca. $2 \mathrm{~cm}$ proximal der distalen Epiphysenfuge liegt, und ein ulnarer Zugang, welcher ca. 2 cm distal der Olekranonapophyse liegt, gewählt. Beim Vorschrieben des Drahtes sollte darauf geachtete werden, dass die elastischen Nägel jeweils 3 Abstützpunkte im Knochen aufweisen und so zur Biegestabilität, axialen Stabilität, Translations- und Rotationsstabilität führen ( $\bullet$ Abb. 5) [12]. Die auftretenden Komplikationen bei der elastisch stabilen intramedullären Nagelung (ESIN) sind in der folgenden • Tab. 1 kurz zusammengefasst.

Eine Ruhigstellung in einer Oberarmgipsschiene ist nicht nötig, kann jedoch zur Analgesie für einige Tage erfolgen. Die Nachbehandlung ist wie bei der konservativen Therapie funktionell. Es sollte sowohl postoperativ eine Stellungskontrolle als auch nach 4 Wochen eine Konsolidierungskontrolle erfolgen. Die Metallentfernung ist bei Konsolidierung sicher nach 5-6 Monaten indiziert.
Komplikationen, die bei einer Unterarmschaftfraktur auftreten können, sind u.a. eine verzögerte Spontankorrektur, eine Zunahme der primären Fehlstellung, die Gefahr einer Refraktur aufgrund von Konsolidierungsstörungen bei typischen Grünholzfrakturen oder bei einer Achsenabweichung $>20^{\circ}$ eine Einschränkung bei der Pro- und Supination. Weiterhin kann aufgrund einer Wachstumsstörung eine vorübergehende Längendifferenz auftreten, die sich im Verlauf jedoch meist ausgleicht.

Nachkontrollen sollten regelmäßig alle 3-4 Wochen bis zur freien Funktion stattfinden, um frühzeitig Komplikationen zu detektieren und behandeln zu können $[16,17]$

\section{Vollständige Unterarmschaftfraktur}

Eine Besonderheit dieser Frakturart ist es, dass sie vor allem bei Kindern älter als 10 Jahre vorkommt. Es ist eine Spontankorrektur von Seit-zu-Seit-Verschiebungen sowie Achsenabweichungen von $<10^{\circ}$ möglich. Eine konservative Therapie kann bei Frakturen mit einer Achsenabweichung $<10^{\circ}$ sowie bei isolierten Frakturen von nur einem Unterarmknochen unterhalb der Korrekturgrenzen oder bei Kleinkindern gewählt werden. Dabei ist ein Oberarmgips für 3-4 Wochen die Therapie der Wahl.

Eine operative Therapie sollte gewählt werden, wenn eine Instabilität aufgrund der Fraktur beider Unterarmknochen besteht oder einer der beiden Knochen eine Grünholzfraktur oder einen Biegungsbruch aufweist. Hier ist besonders auf Rotationsfehler, Achsenabweichungen 
- Tab. 1 Komplikationen bei elastisch stabiler intramedullärer Nagelung (ESIN). Quelle: von Laer L. Methodenabhängige Komplikationen. In: Wirth C, Mutschler W, Neu J, Hrsg. Komplikationen Kompakt: Orthopädie und Unfallchirurgie. Stuttgart: Thieme; 2015.

\begin{tabular}{|l|l|l|}
\hline Komplikation & vermeidbar & Prophylaxe \\
\hline $\begin{array}{l}\text { sekundäre Achsenabweichung } \\
\text { falsche Indikation zur ESIN }\end{array}$ & $\begin{array}{l}\text { ESIN für längsstabile Frakturen } \\
\text { Abwarten, ggf. Refrakturierung, } \\
\text { Korrekturosteotomie }\end{array}$ \\
\hline Rotationsabweichung & $\begin{array}{l}\text { mangelhafte Torsionskontrolle } \\
\text { intraoperativ }\end{array}$ & $\begin{array}{l}\text { intraoperative Überprüfung der } \\
\text { Achse und Torsion klinisch }\end{array}$ \\
\hline Infekt & mangelhafte Hygiene & $\begin{array}{l}\text { korrekte Hygiene, effizientes } \\
\text { Operieren }\end{array}$ \\
\hline Refrakturen & zu frühe ME & $\begin{array}{l}\text { korrekte Technik, Belassen des } \\
\text { Metalls bis zur gesicherten Kon- } \\
\text { solidierung }\end{array}$ \\
\hline Hautirritation & & $\begin{array}{l}\text { Reosteotomie } \\
\text { ausreichende Kürzung des ESINS, } \\
\text { Schutzkappe }\end{array}$ \\
\hline Sehnenschäden & unzureichend gekürzter ESIN & ESIN subkutan belassen \\
\hline ME: Metallentfernung & zu stark gekürzte ESIN & ME \\
\hline
\end{tabular}

a

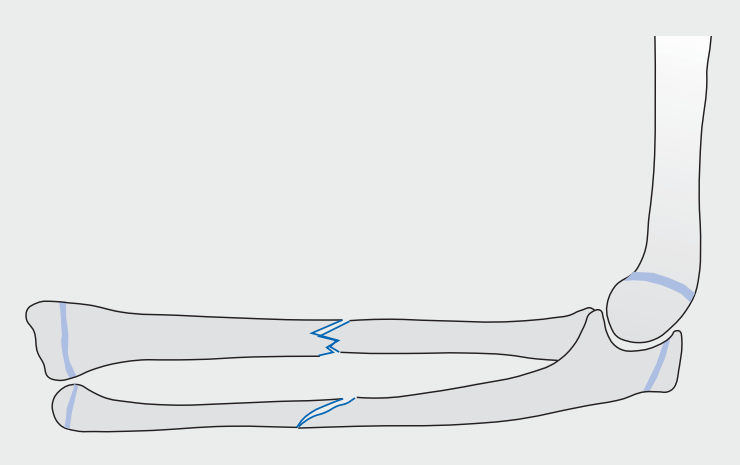

C

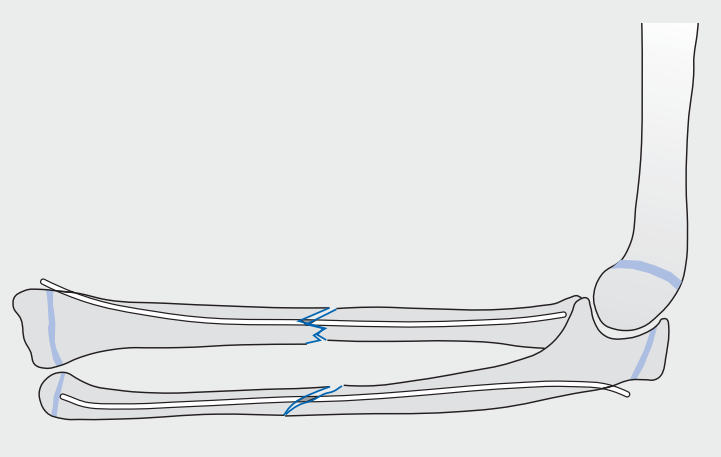

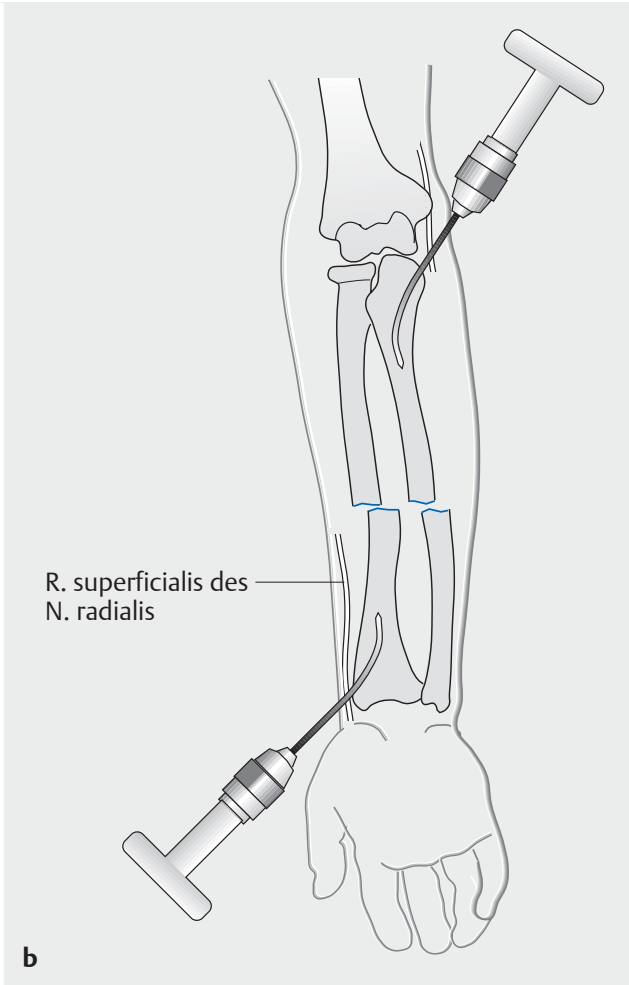

- Abb. 4 a-c Prinzip der elastischen Marknagelung. Quelle: Grützner P, Kattner H. Spezielle Instrumente und Implantate. In: Ewerbeck V, Wentzensen A, Grützner P, Holz F, Krämer KL, Pfeil J, Sabo D, Hrsg. Standardverfahren in der operativen Orthopädie und Unfallchirurgie. 4. Aufl. Stuttgart: Thieme; 2014.

$>10^{\circ}$, Verkürzungen und Interposition von Weichteilen zu achten. Die operative Therapie beinhaltet dabei in aller Regel die geschlossene, bei Repositionshindernis (z. B. Periost- oder Muskelinterponat) ggf. die offene Reposition und Osteosynthese mittels ESIN (s. oben). Sollte es sich bei der Fraktur um eine Mehrfragment- bzw. Trüm- merfraktur, bei denen die Länge mittels ESIN nicht zu halten ist, oder um offene bzw. gelenknahe Frakturen handeln, dann sollte eine Osteosynthese mittels Fixateur externe erwogen werden. Dies ist aber bei Schaftfrakturen, soweit möglich, eher zu vermeiden. Einen Sonderfall 


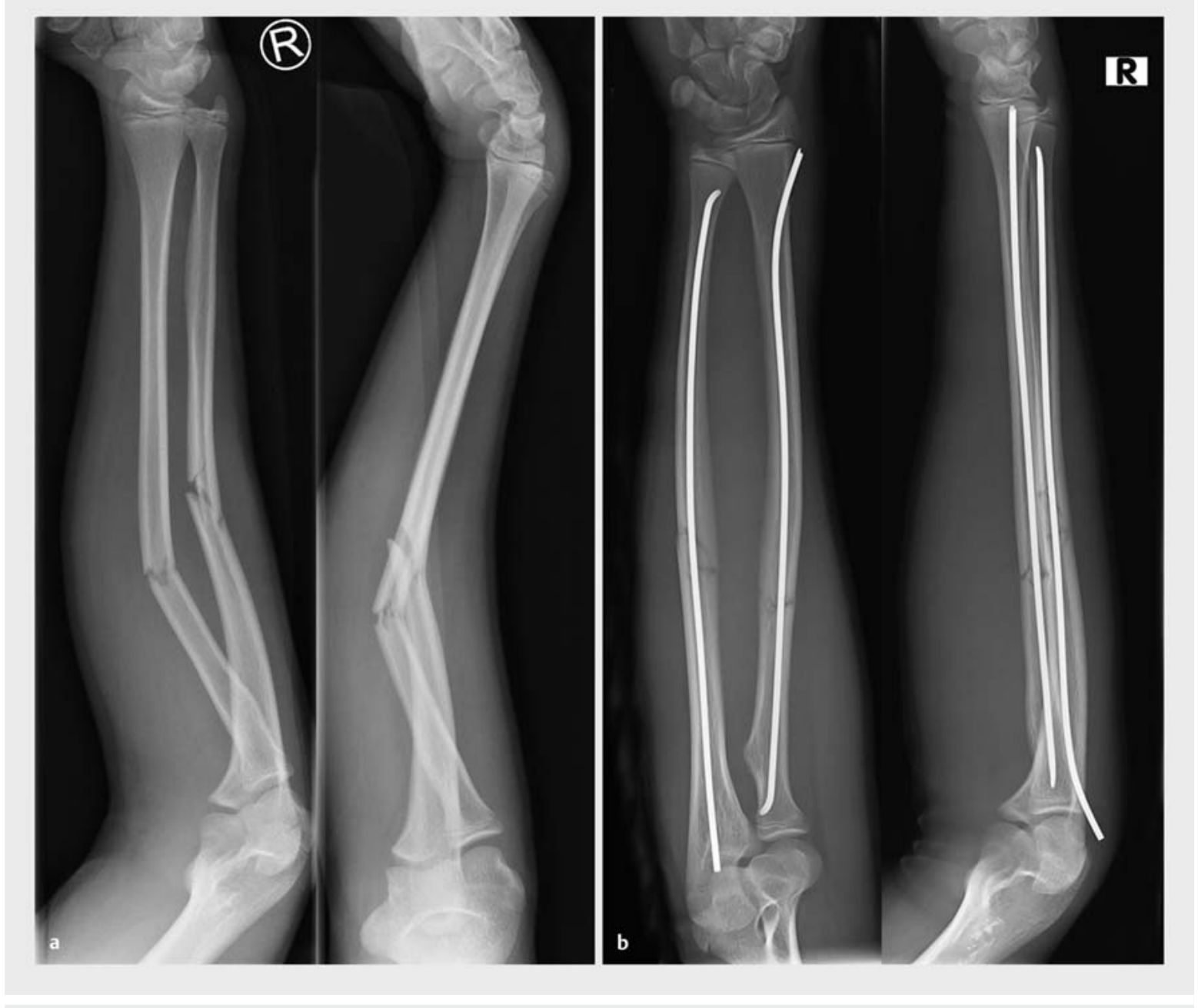

- Abb. 5 a Die konventionelle Röntgendarstellung des Unterarms zeigt eine komplette Unterarmschaftfraktur bei einem 12-jährigen Jungen. b Osteosynthetische Versorgung des Radius und der Ulna mittels elastisch stabiler intramedullärer Nagelung.

stellt die Pseudarthrose bzw. Korrekturosteotomie dar. Hier kann ggf. eine Plattenosteosynthese hilfreich sein.

Als relevante Komplikation kann es bei Achsenabweichungen $>20^{\circ}$ zu Einschränkungen bei der Pro- und Supination kommen. Außerdem kann es zu einer Pseudarthrosenbildung, Synostosenbildung oder einer Refraktur bei früher Metallentfernung ( $<3$ Monate) kommen. Wachstumsstörungen können zu einer vorübergehenden Längendifferenz und Störung der Umwendbewegung führen $[12,13]$.

\section{Monteggia-Verletzung (Ulnafraktur mit Radiuskopfluxation)}

Bei der Monteggia-Verletzung handelt es sich um eine Kombinationsverletzung aus Ulnafraktur und Luxation des Radiusköpfchens. Besonders die Radiuskopfluxation wird häufig bei der Primärversorgung übersehen bzw. das Vollbild der Monteggia-Läsion mangels Erkennen der
Ulnafraktur oder des Ulnabowings. Daher ist immer bei einer Fraktur des Unterarms mit Verkürzung ein korrektes Röntgenbild des Ellenbogens in 2 Ebenen und des Handgelenkes anzufertigen. Ziel der Therapie sollte u.a. die Reposition des Radiusköpfchens sein.

Hierzu kommt es i.d. R. durch die Korrektur zur Ulnafehlstellung bzw. -verkürzung. Es ist dabei zwingend notwendig, eine Kongruenz im proximalen Radioulnargelenk herzustellen, um spätere Einschränkungen bei Umwendbewegungen zu vermeiden. Eine Spontankorrektur im Verlauf ist nicht möglich, sondern der Radiuskopfvorschub verschlimmert sich (s.a. Mörs et al. OP-Journal gleiches Heft).

Eine Indikation zu einer konservativen Therapie mittels geschlossener Reposition kommt nur bei unverschobenen Brüchen infrage. Auch in diesem Fall ist eine Stel- 


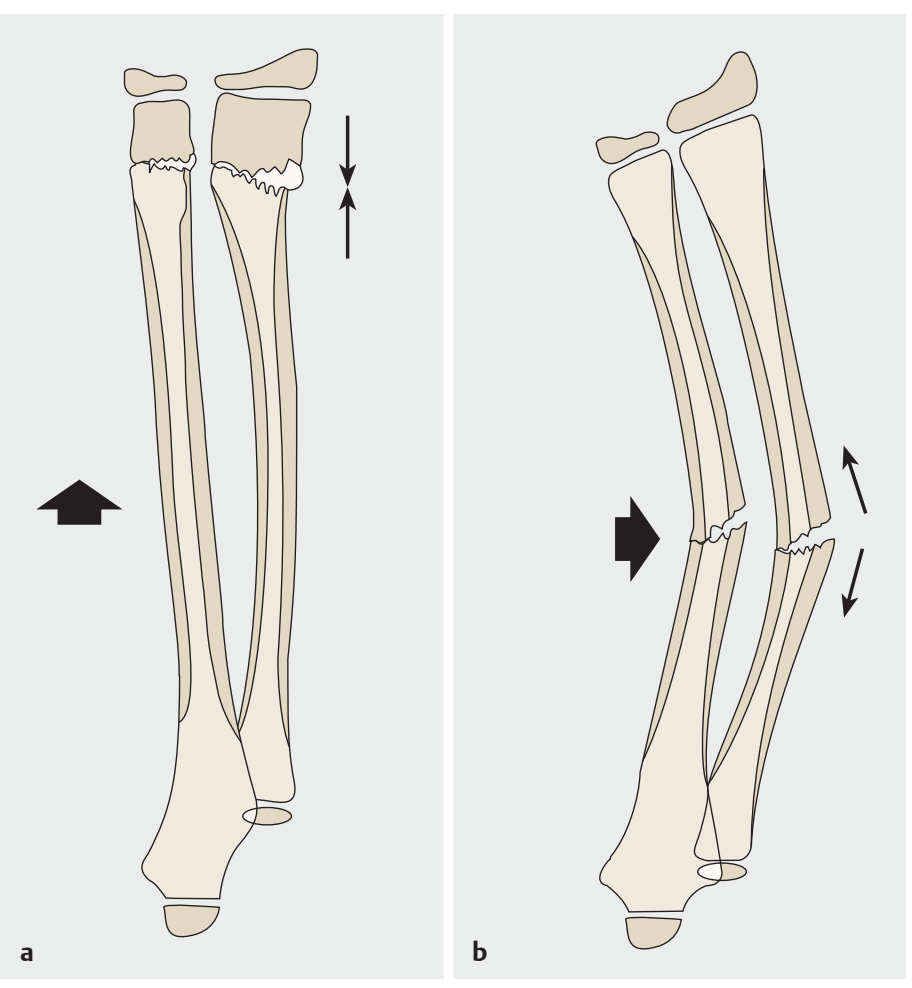

- Abb. 6 a Stauchungsfraktur Unterarmschaft distal metaphysär. b Schema einer diaphysären Grünholzfraktur (Biegungsbruch) am Unterarmschaft. Quelle: von Laer L, Kraus R, Linhart W. Klassifikation und Einteilung von Frakturen im Wachstumsalter. In: von Laer L, Kraus R, Linhart W, Hrsg. Frakturen und Luxationen im Wachstumsalter. 6. Aufl. Stuttgart: Thieme; 2012.

lungskontrolle nach 5 Tagen und eine Konsolidierungskontrolle nach 4 Wochen nötig.

Bei instabilen Ulnafrakturen im Schaftbereich oder instabilen Frakturen ist jedoch eine operative Therapie nicht zu vermeiden. Diese ist nötig, um eine stabile Ulnaosteosynthese mit korrekter Länge zu erlangen. Häufig kommt es bei der Reposition der Ulnafraktur zu einer spontanen Reposition der Radiuskopfluxation. Je nach Art der Ulnafraktur kommt bei einer Schaftfraktur die Osteosynthese mittels ESIN oder auch mal eine Platte infrage. Bei einer metaphysären Fraktur kann eine Zuggurtung notwendig sein oder auch bei Schaftfrakturen ein Fixateur externe. Die Nachbehandlung richtet sich nach dem jeweiligen Osteosyntheseverfahren. Bei der K-Draht-Osteosynthese sollte eine Ruhigstellung für 4 Wochen im Oberarmgips erfolgen. Eine Metallentfernung ist nach Konsolidierungskontrolle nach 8-12 Wochen möglich. Die am häufigsten vorkommende Komplikation bei dieser Art von Fraktur ist das Übersehen der Radiusköpfchenluxation oder bei suboptimaler Ulnalänge und Ulnaangulation auch die Redislokation des Radiuskopfes.

Weiter mögliche Komplikationen sind Schädigungen von N. radialis profundus/N. ulnaris, Radiuskopfnekrosen, Re- luxationstendenz sowie persistierende Bewegungseinschränkungen des Ellenbogens und des Unterarms. Auch Wachstumsstörungen am proximalen Radiusende in Form von Kopfumbaustörungen mit Verplumpung des proximalen Radiusendes können auftreten. Diese können möglicherweise im Verlauf zu Einschränkungen der Pround Supination führen. Diese sind teilweise schwer von angeborenen Fehlbildungen zu unterscheiden, wie solche auch eine akute Luxation vortäuschen können.

\section{Metaphysärer Wulstbruch, Stauchungsfraktur und Grünholzfraktur}

Die Besonderheit von Wulst- oder Stauchungsbrüchen liegt in der beidseits erhaltenen, aber komprimierten Kortikalis (s. - Abb.6a). Es handelt sich dabei um eine stabile Fraktur. Bei der Grünholzfraktur ist dagegen nur eine Kortikalis erhalten, die andere ist durchgebrochen (s. - Abb. 6b). Auch hier handelt es sich um eine stabile Fraktur, die jedoch zu einem erheblichen Fehlwachstum führen kann, da nur die gebrochene Kortikalis eine Kallusreaktion zeigt.

Besonders Wulstbrüche treten fast nur bei kleinen Kindern auf. Gekennzeichnet sind Wulst- und Stauchungsbrüche durch einen erhaltenen Periostschlauch, der praktisch immer zu einer Spontankorrektur führt. Die führende Therapie bei einem Wulst- oder Stauchungsbruch ist die konservative mit einer Ruhigstellung für 2-3 Wochen in einer Unterarmgipsschiene; 3-4 Wochen Ruhigstellung in einer Unterarmgipsschiene sind bei Grünholzbrüchen nötig. Eine Röntgenkontrolle ist bei einem Wulst- oder Stauchungsbruch i.d.R. nicht nötig. Eine Kontrolle auf Druckschmerzhaftigkeit reicht meist im Verlauf als diagnostisches Mittel aus. Bei Grünholzfrakturen sollte nach 5-8 Tagen eine Stellungskontrolle und nach 4 Wochen eine Konsolidierungskontrolle mittels Röntgenuntersuchung erfolgen, um bei zunehmendem Fehlwachstum noch eine operative Korrektur erwägen zu können $[12,13]$.

Die Indikation zur operativen Versorgung ist fast nur bei den Grünholzfrakturen zu stellen, die zu einem Fehlwachstum führen können. Auch dies ist altersabhängig, kann aber auch bei kleinen Kindern noch notwendig sein. Bei Achsenabweichungen im Alter unter 8 Jahren $>30^{\circ}$, über 8 Jahren $>25^{\circ}$ und ab 12 Jahren bei $10^{\circ}$ ist eine operative Versorgung nötig. Hierbei wird die inkomplette Fraktur in eine komplette überführt. Im selben Eingriff ist dann auch eine osteosynthetische Versorgung nötig, die in solchen Fällen mit einer Plattenosteosynthese erfolgt $[11,12,15]$.

Im Verlauf können sowohl bei der konservativen als auch bei der operativen Variante Komplikationen wie z.B. Wachstumsstörungen auftreten. Eine Bewegungseinschränkung ist nur selten der Fall. Wenn es jedoch dazu kommt, entsteht sie aufgrund einer Fehlverheilung des 
distalen Radius und der Ulna am ehesten im distalen Radioulnargelenk (DRUG). Zu einer Wachstumsstörung kommt es in erster Linie durch einen vorzeitigen partiellen oder totalen Fugenverschluss. Hier ist dann eine Verlängerungsosteotomie des Radius zur Wiederherstellung der Länge indiziert. In seltenen Fällen kann es zu einem vorübergehenden Mehrwachstum (vor allem des Radius) kommen, der sich jedoch im Verlauf des weiteren Wachstums wieder ausgleicht [12].

\section{Vollständige Fraktur des distalen Unterarms}

Bei dieser Art von Fraktur kommt es häufig zu einer erheblichen Schwellung und Schmerzhaftigkeit. Spontankorrekturen sind bei Kindern < 12 Jahren bis $30^{\circ}$ in der Sagittalebene und bis zu $10^{\circ}$ in der Frontalebene prinzipiell möglich. Bei kleineren Kindern ist sogar eine höhergradige Korrektur möglich. Ab einem Alter von 12 Jahren sind Spontankorrekturen von Achsenfehlstellungen nicht mehr ausreichend möglich. Die Indikation zur konservativen Therapie wird heutzutage kaum noch gestellt, da meist eine Reposition erforderlich ist und diese bei Kindern zum einen in Vollnarkose bzw. Analgosedierung durchgeführt wird und zum anderen diese durch eine Osteosynthese mit K-Draht problemlos und stabil durchgeführt werden kann. Sollte man sich dennoch für eine konservative Therapie entscheiden, ist eine Ruhigstellung für 4 Wochen in einer Oberarmgipsschiene nötig. Die Nachbehandlung sollte funktionell erfolgen mit einer Stellungskontrolle am 8. Tag und einer Konsolidierungskontrolle nach 4 Wochen $[12,13]$.

Bei einer Seit-zu-Seit-Verschiebung von mehr als einem Viertel der Schaftbreite bzw. bei einer Achsenabweichung $>30^{\circ}$ (Kleinkind) und $10^{\circ}$ (12-Jährige) in der Sagittalebene und $>10^{\circ}$ in der Frontalebene sowie bei Rotationsfehlern und instabilen Frakturen ist eine operative Therapie nötig. Weiterhin sollte bei Flexionsfrakturen des Radius, Verkürzungen des Radius $>5 \mathrm{~mm}$, begleitenden Gefäß-Nerven-Verletzungen und Pseudarthrosen immer eine operative Versorgung gewählt werden.

Hierbei erfolgt die Reposition in Vollnarkose bzw. Analgosedierung und die sichere Stabilisierung durch eine KDraht-Osteosynthese. In den meisten Fällen handelt es sich um eine geschlossene Reposition, die mittels des Kapandji-Verfahrens oder transepiphysär über den Processus styloideus radii durchgeführt wird. Bei der Kapandji-Spickung handelt es sich um ein Verfahren, bei dem 2 K-Drähte von proximal-dorsal in den Frakturspalt eingeführt werden und durch Hebeln eine indirekte Aufrichtung der Fraktur erreicht wird, ohne die Epiphyse zu tangieren (s. - Abb. 7 und 8). Bei der Alternative über den Processus styloideus radii muss darauf geachtet werden, dass man die Fuge nicht mehrmals überbohrt, da es dadurch zu einem vorzeitigen Fugenschluss kommen kann [12].

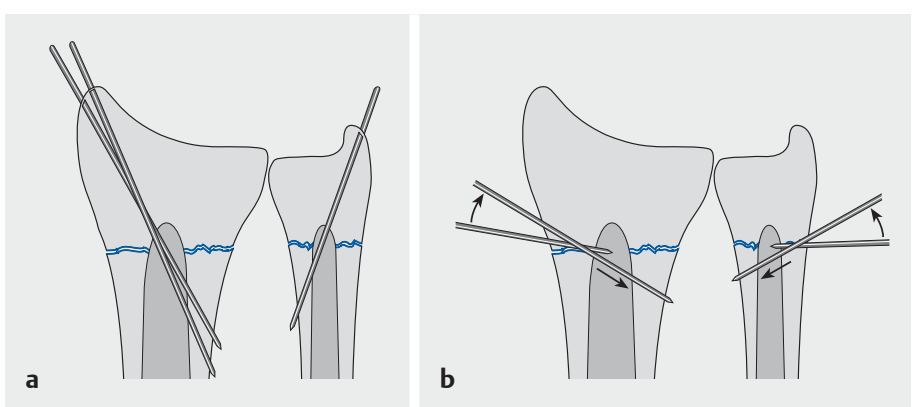

- Abb. 7 a Klassische Kirschner Draht-Osteosynthese bei Radiusfraktur. b Kirschner-Draht-Osteosynthese nach Kapandji von dorsal oder radial. Quelle: Grote S. Knöcherne Verletzungen am Handgelenk. In: Wirth C, Mutschler W, Kohn D, Pohlemann T, Hrsg. Praxis der Orthopädie und Unfallchirurgie. 3. Aufl. Stuttgart: Thieme; 2013.

Bei Adoleszenten kann bei bereits begonnenem Fugenschluss und kräftigen Armen über eine Osteosynthese mittels T-Platte nachgedacht werden (s. - Abb. 9). Handelt es sich bei der Fraktur um eine instabile Flexionsfraktur, wird häufig ein palmarer Zugang über den M. flexor carpi radialis mit einer radialen Ablösung des M. pronator quadratus und einer anatomieorientierten Osteosynthese mittels palmarer Abstütz-T-Platte präferiert [11,12].

In allen vorgestellten Fällen ist die Ruhigstellung in einem Oberarmgips für 2 Wochen, mit anschließend weiterer Ruhigstellung für 2 Wochen in einem Unterarmgips nötig. Weiterhin ist postoperativ eine Stellungskontrolle und nach 4 Wochen eine Konsolidierungskontrolle nötig. Die Metallentfernung kann nach 4-6 Wochen angestrebt werden. Komplikationen, die im Rahmen der vollständigen Fraktur des distalen Unterarms auftreten können, sind Kompartmentsyndrom, Pseudarthrosen der Processus styloidei radii et ulnae, Wachstumsstörungen in Form einer vorübergehenden Längendifferenz oder eine Fehlstellung aufgrund eines vorzeitigen partiellen oder totalen Fugenschlusses [12]. Typische Komplikationen bei der K-Draht-Osteosynthese sind in > Tab. 2 noch einmal aufgeführt.

\section{Epiphysenfugenverletzungen des distalen Unterarms}

Frakturen im Bereich der Epiphyse stellen eine Rarität im Bereich des distalen Unterarms dar. Jedoch ist der distale Radius die häufigste Lokalisation für Epiphyseolysen, die nicht als Gelenkverletzungen gelten, sondern zu den Schaftfrakturen gezählt werden [7, 12]. Im klinischen Alltag werden die Frakturen der Epi- und Metaphyse nach der Klassifikation von Salter-Harris unterschieden ( $\mathbf{A b b}$. 10). Hierbei unterscheidet man:

- Typ I: Epiphyseolyse ohne Fraktur

- Typ II: metaphysäre Fraktur mit partieller Epiphyseolyse 

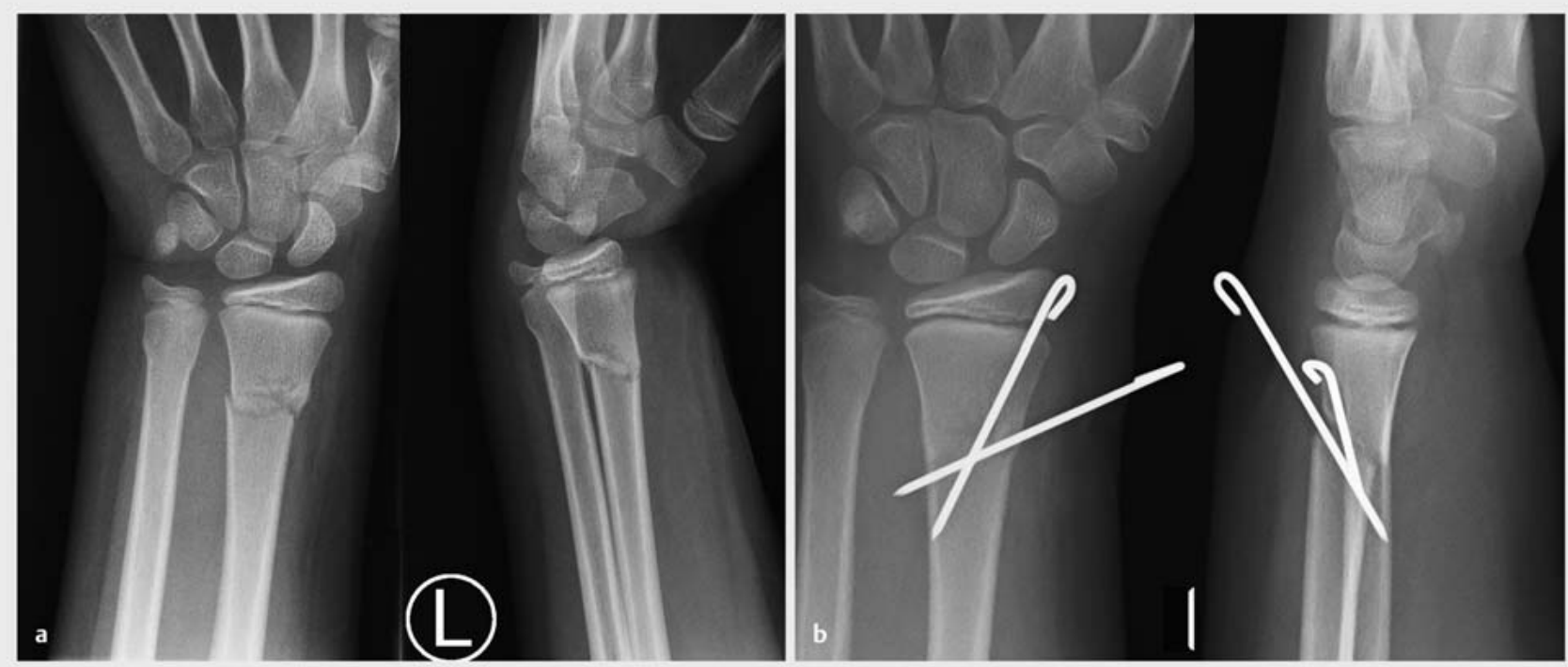

- Abb. 8 a Die konventionelle Röntgendarstellung des Unterarms zeigt eine distale Radiusfraktur mit Achsenabweichung über $10^{\circ}$ bei einem 9 jährigen Mädchen. b Osteosynthetische Versorgung des Radius mittels 2 Kirschner-Drähten.
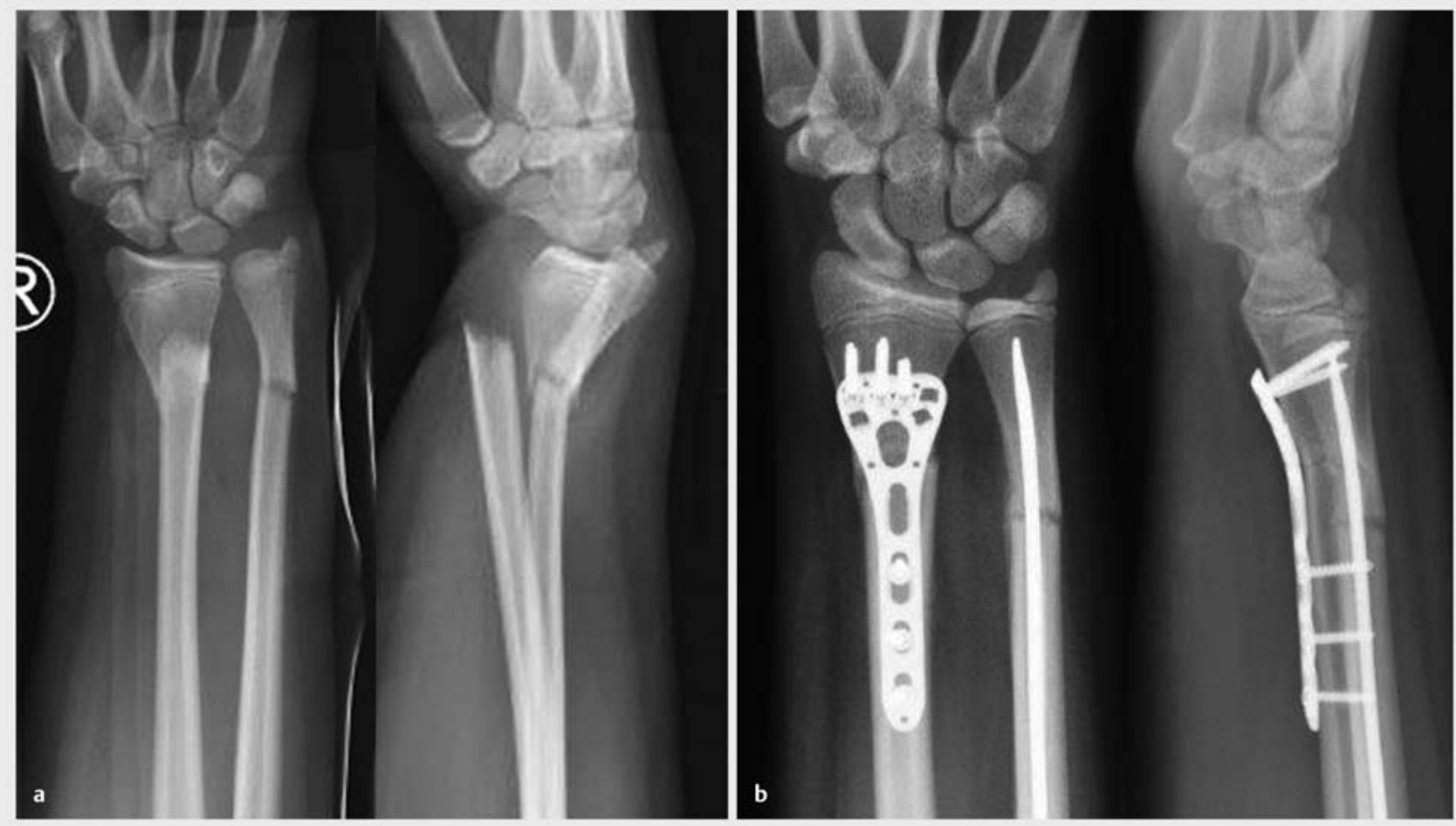

- Abb. 9 a Die konventionelle Röntgendarstellung des Unterarms zeigt eine komplette diametaphysäre Unterarmfraktur bei einem 14-jährigen Mädchen. b Osteosynthetische Versorgung des Radius mittels einer T-Platte und der Ulna mittels einer elastisch stabilen intramedullären Nagelung. 
- Tab. 2 Komplikationen bei der Kirschner-Draht-Osteosynthese. Quelle: von Laer L. Methodenabhängige Komplikationen. In: Wirth C, Mutschler W, Neu J, Hrsg. Komplikationen Kompakt: Orthopädie und Unfallchirurgie. Stuttgart: Thieme; 2015.

\begin{tabular}{|l|l|l|l|}
\hline Komplikation & möglichst zu vermeiden & Prophylaxe & Therapiemöglichkeit \\
\hline Infekt & $\begin{array}{l}\text { mangelhafte Hygiene, häufige Bohr- } \\
\text { versuche }\end{array}$ & gezielte Spickung & lokale Infektsanierung, ME \\
\hline Nervenschäden & $\begin{array}{l}\text { iatrogene Durchtrennung, K-Draht- } \\
\text { Durchbohrung }\end{array}$ & gezielte Spickung (ggf. offen) & ME und Spontanverlauf abwarten \\
\hline Sehnenschäden & $\begin{array}{l}\text { iatrogene Durchtrennung, K-Draht- } \\
\text { Durchbohrung, Sekundärschäden } \\
\text { bei versenktem und nicht umge- } \\
\text { bogenen Draht }\end{array}$ & gezielte Spickung (ggf. offen) & $\begin{array}{l}\text { ME und eventuell Versorgung der } \\
\text { Läsion }\end{array}$ \\
\hline Metallbruch & $\begin{array}{l}\text { Überdrehung/Verwringen geboge- } \\
\text { ner K-Drähte }\end{array}$ & senkrechtes Bohren & meist Belassen der Drähte \\
\hline $\begin{array}{l}\text { Zweiteingriff zur ME we- } \\
\text { gen versenktem K-Draht }\end{array}$ & $\begin{array}{l}\text { Versenken des K-Drahtes unter das } \\
\text { Hautniveau }\end{array}$ & $\begin{array}{l}\text { K-Draht oberhalb des Hautniveaus } \\
\text { belassen }\end{array}$ & $\begin{array}{c}\text { Draht mit gipsfreiem Hof umge- } \\
\text { ben }\end{array}$ \\
\hline ME: Metallentfernung & & & \\
\hline
\end{tabular}

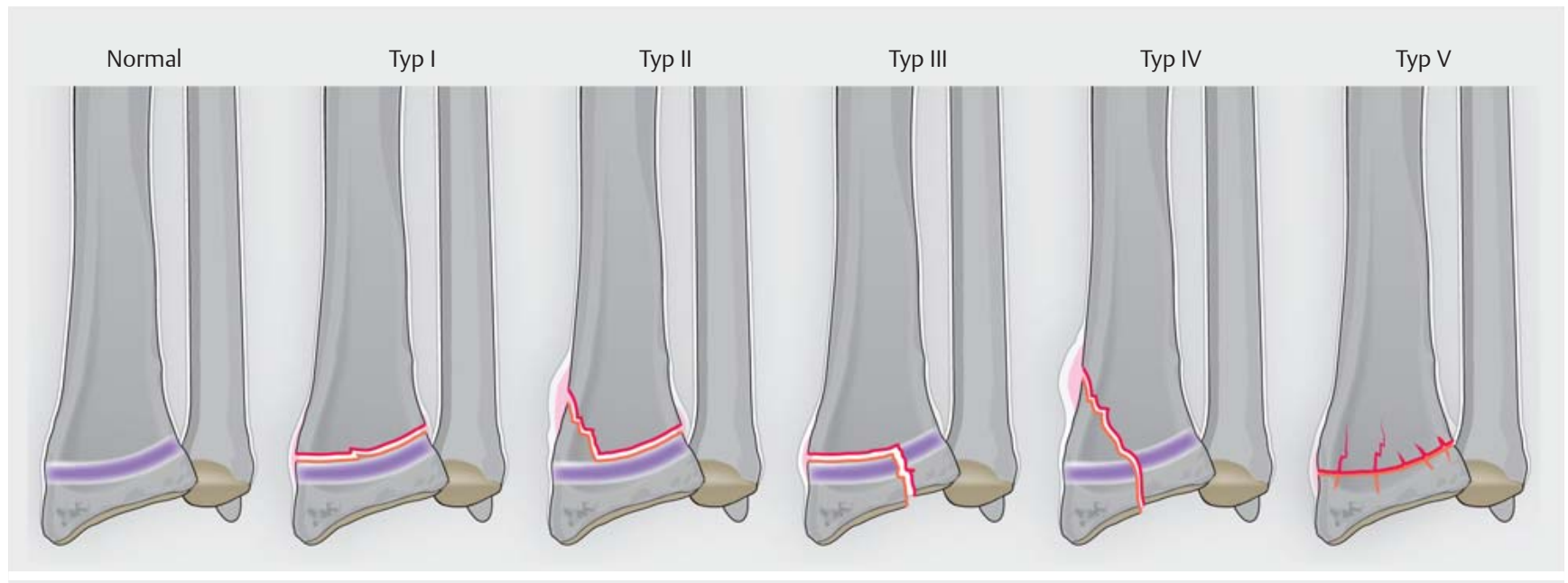

- Abb. 10 Salter-Harris-Klassifikation für Frakturen der Epi- und Metaphyse.

- Typ III: epiphysäre Fraktur mit partieller Epiphyseolyse

- Typ IV: epi- und metaphysäre Fraktur

- Typ V: axiale Stauchungsfraktur der Epiphysenfuge

Trotz der häufig erheblichen Fehlstellung im Bereich der Epiphyse ist oft ein gutes Korrekturpotenzial vorhanden. Diese Korrekturmöglichkeit sowie die Behandlungsalternativen sollten jedoch im Vorfeld ausführlich mit den Eltern besprochen werden. Die möglichen Spontankorrekturgrenzen liegen bei Kindern < 12 Jahre bei bis zu $30^{\circ}$ in der Frontalebene und bis zu $10^{\circ}$ in der Sagittalebene. Bei Kindern, die älter als 12 Jahre sind, ist kaum eine Spontankorrektur mehr vorhanden, weshalb eine achsengerechte Stellung angestrebt werden sollte. Eine konservative Therapie ist möglich bei Frakturen innerhalb der Korrekturgrenzen [11-13]
Im Gegensatz dazu sind die Frakturen vom Typ SalterHarris III/IV und bei Übergangsfrakturen (Dislokation $<2 \mathrm{~mm}$ ) echte Gelenkfrakturen, die auch den Gelenkknorpel betreffen. Liegt hier die Dislokation im Gelenkspalt > $2 \mathrm{~mm}$, ist eine operative Versorgung prinzipiell indiziert. Hierbei wird eine geschlossene Reposition und eine definitive Versorgung mittels K-Draht-Osteosynthese bevorzugt [12].

\section{Galeazzi-Verletzung}

Von einer Galeazzi-Verletzungen spricht man bei einer dislozierten Radiusfraktur in Kombination mit einer Ulnakopfluxation. Diese Art von Verletzung kommt sehr selten vor, vor allem aber bei Kindern, die älter als 10 Jahre sind. Ziel jeder Therapieform ist die Reposition und Stabilisierung des Radius mit dadurch indirekt hervorgebrachter Korrektur der Inkongruenz im distalen Radioulnarge- 
lenk. Eine konservative Therapie mittels geschlossener Reposition ist nur bei unverschobenen Brüchen möglich.

Eine Indikation zur operativen Therapie ist gegeben, wenn es zu einer Achsenabweichung des Radius von $>10^{\circ}$, zu einer Verkürzung des Radius oder zu einer Interposition von Weichteilen (z.B. Sehnen) kommt. Je nach Frakturtyp kann die operative Therapie mittels ESIN, Fixateur externe, K-Drähten oder in seltenen Fällen mittels Plattenosteosynthese erfolgen. Zur Einstellung des distalen Radioulnargelenks sollte postoperativ eine Ruhigstellung für 3 Wochen in Supination in einer Oberarmgipsschiene erfolgen. Eine Stellungskontrolle ist postoperativ sowie 2 Wochen nach der operativen Therapie nötig, eine Konsolidierungskontrolle sollte nach 4 Wochen erfolgen. Je nach gewähltem Osteosyntheseverfahren ist die Metallentfernung bei K-Drähten und Fixateur externe nach 4 Wochen, bei ESIN nach 3-4 Monaten und bei der Plattenosteosynthese nach 8-12 Wochen nach vorheriger Konsolidierungskontrolle möglich. Komplikationen im Bereich des DRUG in Form von Instabilität mit rezidivierenden Luxationen oder Subluxationsstellung, Einschränkung von Pro- und Supination, chronische Schmerzen oder Kraftverlust sind möglich. Weiterhin kann es zu Wachstumsstörungen kommen, die zu chronischen Beschwerden im DRUG führen. Ist dies der Fall, ist eine MRT-Funktionsdiagnostik, ggf. Arthroskopie des Handgelenkes, zur Einschätzung der Verletzung nötig. Sollten sich Wachstumsstörungen zeigen, gibt es die Möglichkeit der späteren Korrektur in Form von Radiusosteotomie und erneuter Osteosynthese im korrekten Längenverhältnis, Ulnaverkürzungsosteotomie oder Radiusverkürzungsosteotomie nach Wachstumsabschluss zur Wiederherstellung der Kongruenz im distalen Radioulnargelenk.

\section{Fazit für die Praxis}

1. Frakturen im Bereich des Unterarms stellen die am häufigsten vorkommende Fraktur im Kindesalter dar.

2. Ziel jeder Behandlung bei Frakturen im Wachstumsalter ist eine kindgerechte Therapie, die mit einem minimalen diagnostischen und therapeutischen Aufwand ein optimales Behandlungsergebnis erreicht.

3. Bei jeder Therapiefindung sollte man die hohe Potenz zur Spontankorrektur aufgrund der Nähe zur Wachstumsfuge und dem hohen Anteil der distalen Fuge am Längenwachstum berücksichtigen.

4. Es können nicht nur Achsenfehlstellungen in allen Ebenen, sondern auch Seit-zu-Seit-Verschiebungen gut korrigiert werden.

5. Eine Korrektur von Rotationsfehlstellungen ist nicht ausreichend möglich.

6. Das Diagnostikum der Wahl ist das Röntgenbild in 2 Ebenen. Es sollte bei Schaftfrakturen darauf geachtet werden immer die angrenzenden Gelenke mit abzubilden, um Begleitverletzungen nicht zu übersehen.
7. Aufgrund des hohen Korrekturpotenzials kann der überwiegende Anteil der Frakturen im Bereich des Unterarms mit nicht invasiven Verfahren behandelt werden.

8. Eine klare OP-Indikation stellen instabile Frakturen, dislozierte (Grünholz-)Frakturen über der Toleranzgrenze, intraartikuläre Frakturen, Gefäß- oder Nervenläsionen, ein ausgeprägter Weichteilschaden, Rotationsfehlstellungen sowie sekundäre Dislokationen dar.

9. Als operative Verfahren stehen u.a. die K-Draht-Osteosynthese, ESIN-Technik und in seltenen Fällen die Plattenosteosynthese bzw. Anlage eines Fixateur externe zur Verfügung.

10. Die Prognose ist nach adäquater Therapie sehr gut und in den überwiegenden Fällen heilt die Fraktur im Kindesalter komplikationslos aus.

\section{Interessenkonflikt}

Der korrespondierende Autor gibt an, dass kein Interessenkonflikt besteht.

\section{Autorinnen/Autoren}

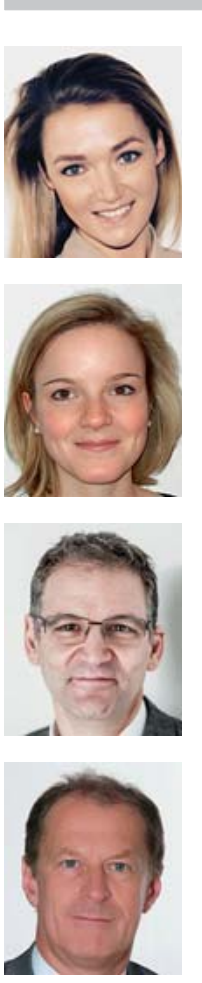

\section{Britta Sundermann}

Dr. med., Assistenzärztin, Klinik für Unfall-, Hand- und Wiederherstellungschirurgie, Universitätsklinikum Frankfurt

\section{Katharina Mörs}

Dr. med., Assistenzärztin, Klinik für Unfall-, Hand- und Wiederherstellungschirurgie, Universitätsklinikum Frankfurt

\section{Johannes Frank}

Prof. Dr. med., Stellvertretender Direktor, Klinik für Unfall-, Hand- und Wiederherstellungschirurgie, Universitätsklinikum Frankfurt

\section{Ingo Marzi}

Prof. Dr. med., Direktor, Klinik für Unfall-, Handund Wiederherstellungschirurgie, Universitätsklinikum Frankfurt

Korrespondenzadresse

\section{Dr. med. Britta Sundermann}

Klinik für Unfall-, Hand- und Wiederherstellungschirurgie Universitätsklinikum Frankfurt

Theodor-Stern-Kai 7

60590 Frankfurt am Main

Tel.: +496963015069

britta.sundermann@kgu.de 


\section{Literatur}

[1] Barrett IR, Bellemore Khosla Set al. Incidence of childhood distal forearm fractures over 30 years: a population-based study. JAMA 2003; 290: 1479-1485

[2] Kraus R, Ploss C, Staub L et al. Fractures of long bones in children and adolescents. Osteo Trauma Care 2006; 14: 39-44

[3] Kraus R, Schneidmüller D, Röder C. Häufigkeiten von Frakturen der langen Röhrenknochen im Wachstumsalter. Dtsch Arztebl 2005; 102: A838-A842

[4] Wilkins KE. Principles of fracture remodeling in children. Injury 2005; 36 (Suppl. 1): A3-A11

[5] Kraus R, Horas U, Szalay G et al. School-related injuries. A retrospective 5-year evaluation. Eur J Trauma Emerg Med 2011; 37: 411-418

[6] Slongo T, Audigé L, Clavert J-M et al. The AO comprehensive classification of pediatric long-bone fractures: a web-based multicenter agreement study. J Pediatr Orthop 2007; 27: 171-180

[7] Schneidmüller D, Röder C, Kraus R et al. Development and validation of a paediatric long-bone fracture classification. A prospective multicentre study in 13 European paediatric trauma centres. BMC Musculoskelet Disord; 2011; 12: 89

[8] Von Laer L, Kraus R, Linhart WE. Korrekturmechanismus des wachsenden Skeletts: In: von Laer L, Kraus R, Linhart WE, Hrsg. Frakturen und Luxationen im Wachstumsalter. Stuttgart, New York: Thieme; 2007: 12-17

[9] Green NE, Swiontkowski MF, eds. Skeletal Trauma in Children. Philadelphia: W.B. Saunders Company; 1998
[10] Rodriguez-Merchán EC. Pediatric fractures of the forearm. Clin Orthop Relat Res 2005; 432: 65-72

[11] Laurer H, Sander A, Wutzler $S$ et al. [Therapy principles of distal fractures of the forearm in childhood]. Chirurg 2009; 80: 1042-1052

[12] Marzi I, Hrsg. Kindertraumatologie. 3. Aufl. Heidelberg: Springer; 2016: 1-572

[13] Von Laer L, Kraus R, Linhart WE, Hrsg. Frakturen und Luxationen im Wachstumsalter. 6. Aufl. Stuttgart, New York: Thieme; 2012

[14] Ackermann O, Liedgens P, Eckert K et al. Sonographische Diagnostik bei metaphysären Wulstbrüchen. Unfallchirurg 2009; 112: 706-711

[15] Lieber ], Sommerfeldt DW. [Diametaphyseal forearm fracture in childhood]. Unfallchirurg 2011; 114: 292-299

[16] Schmittenbecher P. Frakturen der oberen Extremität im Kindes-/Wachstumsalter. Chirurg 2017; 88: 451-466

[17] Aidelsburger P, Grabein K, Huber A et al. Die elastisch stabile intramedulläre Nagelung bei instabilen kindlichen Unterarmschaftfrakturen. Deutsche Agentur für Health Technology Assessment des Deutschen Instituts für Medizinische Dokumentation und Information (DAHTA@DIMDI), Hrsg. 2006: 1-163; Im Internet: https://portal.dimdi.de/de/hta/hta_berichte/ hta121_bericht_de.pdf; Stand: 10.09.2018

Bibliografie

DOI https://doi.org/10.1055/a-0623-7069

OP-JOURNAL 2018; 34: 290-301 @ Georg Thieme Verlag KG Stuttgart · New York ISSN 0178-1715 